\title{
Separate and combined effects of hypobaric hypoxia and hindlimb suspension on skeletal homeostasis and hematopoiesis in mice
}

This article was published in the following Dove Press journal: Hypoxia

\author{
Marjorie Durand' \\ Jean-Marc Collombet' \\ Sophie Frasca' \\ Véronique Sarilar ${ }^{2}$ \\ Jean-Jacques Lataillade ${ }^{1,3, *}$ \\ Marie-Caroline Le Bousse- \\ Kerdilès ${ }^{4, *}$ \\ Xavier Holy ${ }^{2, *}$ \\ 'Department of Medical and Surgical \\ Assistance to the Armed Forces, French \\ Forces Biomedical Research Institute \\ (IRBA), Brétigny sur Orge, Cedex 9/223, \\ France; ${ }^{2}$ Department of Platforms \& \\ Technological Research, French Armed \\ Forces Biomedical Research Institute \\ (IRBA), Brétigny sur Orge, Cedex, 91223, \\ France; ${ }^{3}$ Unit for Research Development, \\ Armed Forces Blood Transfusion Center, \\ Clamart, Cedex 9214I, France; ${ }^{4}$ INSERM \\ UMR-S/MDI 197, Hôpital Paul Brousse, \\ Villejuif, Cedex 94807, France
}

*These authors contributed equally to this work
Correspondence: Marjorie Durand Department of Medical and Surgical Assistance to the Armed Forces, French Forces Biomedical Research Institute (IRBA), BP 73, Brétigny sur Orge, Cedex 91223 , France

Tel +33 I7 $865 \quad$ II 46

Fax +33 $17865 \quad 1646$

Email marjorie-durand@hotmail.fr
Purpose: Bone marrow response to an organismal stress is made by orchestrating the interplay between hematopoietic stem/progenitor cells (HSPCs) and mesenchymal stromal cells (MSCs). Neither the cellular nor the molecular factors that regulate this process are fully understood, especially since this mechanism probably varies depending on the type of stress. Herein, we explored the differentiation and fate of MSCs and HSPCs in mice challenged with a hematopoietic stress or a mechanical stress applied separately or in combination.

Methods: Mice were subjected to 4 days of hypobaric hypoxia (hematopoietic challenge) and/or 7 days of hindlimb suspension (stromal challenge) and then sacrificed for blood and bone collection. Using hematological measurements, colony-forming unit assays, bone histomorphometry and array-based multiplex ELISA analysis, we evaluated challenge influences on both MSC and HSPC mobilization, differentiation (osteoblasts, osteoclasts, and mature blood cells) and fate.

Results: We found that hypoxia leads to HSPC mobilization and that an imbalance between bone formation and bone resorption accounts for this mobilization. Whilst suspension is also associated with an imbalance between bone formation and bone resorption, it does not induce HSPC mobilization. Then, we revealed cellular interactions by combining hematopoietic and stromal challenges together in mice. We showed that the hypoxia-driven HSPC mobilization is moderated by suspension. Moreover, when applied in a hypoxic environment, suspension offsets bone imbalance. We identified stroma cell-derived factors MIP-1 $\alpha$, HGF and SDF-1 as potent molecular key players sustaining interactions between hindlimb suspension and hypobaric hypoxia.

Conclusion: Taken together, our data highlight the benefit of combining different types of stress to better understand the interplay between MSCs and HSPCs.

Keywords: hypobaric hypoxia, hindlimb suspension, mesenchymal stromal cells, hematopoietic stem and progenitor cells

\section{Introduction}

Musculoskeletal and hematopoietic systems display great plasticity and are both able to adapt in response to changes in work demand. Coordinated response to environmental stress is made, at least in part, by the interplay between mesenchymal stromal cells (MSCs) and hematopoietic stem and progenitor cells (HSPCs) in bone marrow (BM) niches. For instance, in oncology, administration of the hematopoietic cytokine granulocyte-colony stimulating factor (G-CSF) induces HSPC 
mobilization from bone marrow leading to MSC pool expansion as a consequence of the G-CSF-driven increased bone turnover. ${ }^{1}$

In high altitude environments, a well-known standard physiological adaptation to hypobaric hypoxia is the increase in red blood cell production. ${ }^{2}$ At the BM level, fine physiological adjustments are made to enhance both erythroid progenitor proliferation and maturation. This process is likely to be orchestrated by the hematopoietic cytokine erythropoietin (EPO), whose production is increased by hypoxia. Beyond its hematopoietic function, a growing body of evidences suggest that EPO might also have a direct action on mesenchymal lineage cells in vivo. ${ }^{3}$ This view is sustained by some studies reporting that bone development is impaired at high altitude and that chronic long-term hypobaric hypoxia might be a potent bone loss trigger. 4,5

However, whether these observations are a consequence of the hematopoietic adaptation to altitude or whether it results from a direct action of the hypoxia signaling on bone cells remains under debate.

What is however well documented is bone cell adaptation to suspension. ${ }^{6}$ Long-term exposure to a weightlessness space environment causes astronaut bone loss. ${ }^{7}$ Due to the limited spaceflight opportunities, the hindlimb unloading model, also known as the hindlimb suspension model, was developed in rodents as a ground-based model of weightlessness to investigate the underlying mechanisms leading to bone loss. ${ }^{8}$ Our team and others reported that bone mass reduction in hindlimb suspended mice is related to a sustained decrease in MSC number and osteoblast differentiation, altogether with a transient increase in the number of HSPC-derived osteoclasts. ${ }^{9-11}$ Aside from bone loss, widespread alterations in the hematopoietic system have been reported after exposure to microgravity and hindlimb suspension, including decreased differentiation of white blood cells from hematopoietic precursors and reduction in red blood cell mass. ${ }^{12,13}$

Due to the aforementioned observations, we sought to take advantage of interactions between hypoxia (hematopoietic stress) and suspension (stromal stress), speculating that combination of both stressors might be relevant to reveal biological synergies or antisynergies leading to a better knowledge of HSPC and MSC maintenance. In other words, our assumption was that stromal and hematopoietic challenges applied separately can both modulate either positively or negatively stem and progenitor cell maintenance, but when applied together one can superimpose or oppose its effects on the other one. Moreover, with the recent focus on deep space exploration and ultimately the shift toward manned planetary exploration to Mars, there is a need for space medicine to assess the combined effects of microgravity-induced unloading and hypoxia on hematological and musculoskeletal functions since future space habitats are expected to cumulate both microgravity drawbacks and reduced $\mathrm{PO}_{2}$ atmosphere. ${ }^{14}$ In this context, the very recent PlanHab (Planetary Habitat Simulation) project sought to address the separate and combined effects of hypoxia and sustained recumbency (bedrest) on human physiological systems, including both the hematopoietic and musculoskeletal ones. ${ }^{15,16}$ In the blood of PlanHab volunteers, an interesting finding was the increase in EPO level under hypoxic conditions and the EPO level reduction induced by bed-rest confinement. In bedridden patients exposed to hypoxia, investigators showed that hypoxia prevents the bed restdriven EPO decrease. ${ }^{16}$

Regarding bone metabolism, PlanHab findings were rather surprising because the study refutes the expected idea of modulatory effects by hypoxia upon bed restinduced bone loss. ${ }^{15}$

However, it is noteworthy that PlanHab study did not examine interactions between hematopoietic and stromal stresses at the bone marrow level. Yet, it can be assumed that any interactions between reduced gravity and low inspired $\mathrm{PO}_{2}$ regarding hematologic and musculoskeletal responses will be easier to reveal in the bone marrow compartment, where both MSCs and HSPCs reside.

Therefore, the aim of the present study was to examine the differentiation and fate of MSCs and HSPCs in both bone marrow compartment and blood of mice subjected to hindlimb suspension and hypoxia applied either separately or in combination. As expected, adding hypoxia to suspension revealed interactions not only at the cellular level on HSPC and MSC mobilization and differentiation but also at the molecular level on key regulator factors of BM niches. In parallel, this work also revealed that hematopoietic stress-induced HSPC mobilization is related to an imbalance between bone formation and bone resorption whereas stromal stress-induced imbalance between bone formation and bone resorption is not necessarily linked to HSPC mobilization. These findings suggest that different pathways are involved in these physiological responses.

\section{Materials and methods}

\section{Animal care}

Ten-week-old male C57BL/6J mice (Janvier labs, Le GenestSaint-Isle, France) were individually housed under standard 
conditions of temperature $\left(22 \pm 1^{\circ} \mathrm{C}\right)$ and hygrometry $(45$ $65 \%$ ) with a $12 \mathrm{hr}: 12 \mathrm{hr}$ light-dark cycle (lights on between 07:00 and 19:00). Mice had free access to water and standard laboratory chow. All experiments were approved by the Institutional Animal Care and Research Advisory Committee of IRBA in accordance with French regulation (government decree $\mathrm{N}^{\circ}$ 2013-118) that proceed from the European union guidelines $(2010 / 63 / \mathrm{EU})$ for the protection of vertebrate animals used for experimental and other scientific purposes. The principal investigators of the study have a license to practice animal experimentations.

\section{Experimental protocol}

Mice were randomly assigned to 4 experimental groups ( $n=10$ per experimental group) as described in Figure 1. At $\mathrm{D}_{0}$, half mice population were placed into hindlimb suspension cages for 7 days, following the procedure previously described by Holy et al. ${ }^{17}$ In this model, the hindlimbs of rodents are elevated with an unloading device to produce a $30-35^{\circ}$ head-down tilt angle, which avoids weight-bearing by the hindquarters. At the third day of suspension, the suspended mice were equally separated into 2 groups. One group was kept under normoxic conditions at $101 \mathrm{kPa}$ (NS group; $\mathrm{FiO}_{2}=21 \%$ ) while the other group of suspended mice was housed for 4 days into a hypoxic chamber at $50 \mathrm{kPa}$ (HS group, $\mathrm{FiO}_{2}=10 \%$ ). The remaining half unsuspended mice population was either housed in normoxic conditions for all the experiment duration (NC group) or was exposed to hypobaric hypoxia for the last 4 days of the experiment (HC group). Of note, suspension and hypoxia onset times were delayed by a 3day gap for ethical reasons. Since both stresses are classified as severe procedures, we did not want to initiate these two procedures simultaneously. In a preliminary investigation, we previously validated hematologic and bone metabolism changes induced by both suspension and hypoxia environmental conditions as well as stress durations. ${ }^{18}$ All animals were sacrificed under isoflurane anesthesia at $D_{7}$ for blood (intracardiac puncture) and femur collections.

\section{Hematological measurements}

Blood was collected through intra-cardiac puncture with $3.8 \%$ citrate $\mathrm{w} / \mathrm{v}$ to avoid blood clotting. Hematological measurements were performed using the MS-9 automatic hematology analyzer (Melet Schloesing Laboratoires, Osny, France) with mouse-specific analysis software.

\section{Colony-forming unit (CFU) assays}

HSPCs were quantified by CFU assay in the BM and blood, as previously described. ${ }^{18}$ Hematopoietic colonies were classified according to standardized morphologic criteria as erythroid progenitors (burst-forming unit-erythroid BFU-E) and myeloid-derived progenitors including CFUgranulocyte (CFU-G), CFU-macrophage (CFU-M) and CFU-granulocyte macrophage (CFU-GM). Freshly isolated BM cells from the same femurs were also evaluated for MSC colony forming unit-fibroblast (CFU-F) assay as previously described. ${ }^{18}$ Giemsa-stained adherent colonies (>50 cells) deriving from MSCs were counted on days $12-14$.

\section{Histomorphometry}

One-day formaldehyde-fixed femurs were successively dehydrated in ethanol and xylene, and then embedded in Technovit $^{\circledR}$ resin. Five-micrometer longitudinal sections were prepared and stained with Masson-Goldner trichrome

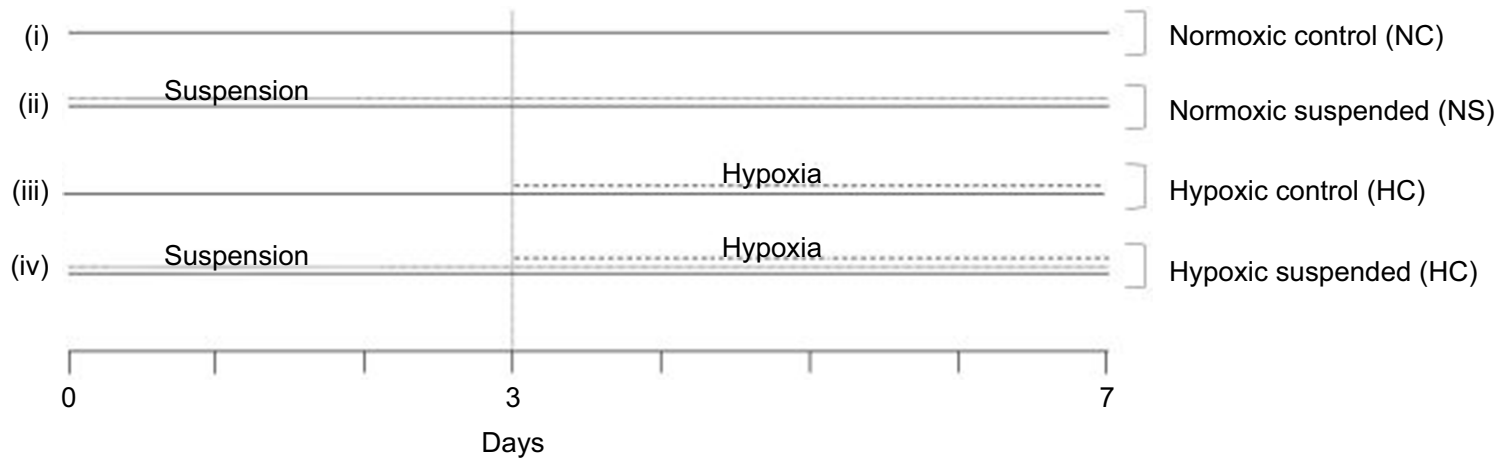

Figure I Experimental set-up. Mice were randomly assigned to 4 different experimental groups ( $\mathrm{n}=10$ mice per group): (i) Normoxic control (NC) group: mice were kept under normoxic conditions $\left(\mathrm{FiO}_{2}=21 \%\right.$ ) from day 0 to the day of sacrifice (day 7); (ii) Normoxic suspended (NS) group: mice were subjected to suspension from day 0 to day 7 under normoxic conditions $\left(\mathrm{FiO}_{2}=21 \%\right.$ ); (iii) Hypoxic control $(\mathrm{HC})$ group: mice were housed into a hypoxic chamber at $50 \mathrm{kPa}(\mathrm{FiO}=10 \%)$ for 4 days before sacrifice $($ day 3 to day 7); (iv) Hypoxic suspended (HS) group: mice were subjected to suspension for 7 days (day 0 to day 7 ) and were transferred into a hypoxic chamber at 50 kPa $\left(\mathrm{FiO}_{2}=10 \%\right)$ for the 4 final days of suspension (day 3 to day 7 ). 
dye. Static bone histomorphometric parameters (osteoblast surface normalized to bone surface Ob.S/BS, osteoclast number normalized to bone surface Oc.N/BS and bone volume/total volume ratio BV/TV) in the secondary metaphyseal area were measured using a Leica Aristoplan microscope under blind conditions. Histomorphometric parameter values were measured in three different nonadjacent sections and averaged.

\section{Protein analysis in BM supernatant and blood plasma}

Levels of different proteins involved in stem cell niche maintenance and progenitor commitment were determined in supernatant of femoral BM flushes using an array-based multiplex sandwich Elisa from RayBiotech (RayBiotech Life, Norcross, GA, USA). Briefly, femurs were flushed with a syringe (26 gauge needle) containing $500 \mu \mathrm{L}$ IMDM $+1 \%$ penicillin-streptomycin-neomycin antibiotic mixture and then $\mathrm{BM}$ flushes were centrifuged for 10 mins at $350 \mathrm{~g}$ and $+4^{\circ} \mathrm{C}$. The resulting supernatants were used for protein analysis. Supernatant samples $(50 \mu \mathrm{L})$ were loaded onto array slides designed with implanted capture antibodies raised against insulin-like growth factor-1 (IGF-1), vascular endothelial growth factor (VEGF), hepatocyte growth factor (HGF), macrophage inflammatory protein-1 $\alpha$ (MIP-1 $\alpha$ ). Fluorescence-based quantification was performed by incubating array slides with HyLite plusTM555-labeled detection antibody and by laser scanner reading with an Agilent G2505B microarray scanning device and Q-analyzer software analysis. This proteome analysis experiment was subcontracted by Tebu-Bio (Tebu-Bio, Le Perray-en-Yvelines, France). SDF-1 (stromal cell-derived factor-1) concentration was assessed by Quantikine enzyme-linked immunosorbent assay following manufacturer's instructions (MCX120 kit, R\&D Systems, Minneapolis, MN, USA) and EPO concentration was measured in plasma using the mouse EPO enzymelinked ELISA kit (MEP00B kit; R\&D Systems, Minneapolis, MN, USA).

\section{Data analysis}

A two-way analysis of variance (ANOVA) was used to elucidate the effects of hypoxia and suspension and their possible interactions (hypoxiaxsuspension) on the outcomes. When interactions in the two-way ANOVA were statistically significant, pairwise comparisons were investigated using Newman Keuls post-hoc test. Whenever interaction was not significant, main effect of hypoxia or suspension was thus reported. Results of statistical tests with $p \leq 0.05$ were considered significant (Statview software, SAS Institute Inc., Cary, NC, USA). All data are expressed as mean \pm standard deviation (SD).

\section{Results}

\section{Suspension alters the hypoxia-induced egress of HSPCs from BM to blood}

We first analyzed the consequence of suspension added onto a hematopoietic stress, namely hypoxia, on the BM hematopoietic progenitor compartment. As shown in Figure 2A, ANOVA underscored a significant effect of hypoxia $(p \leq 0.001)$ by decreasing the number of myeloidderived progenitors (CFU-G+CFU-GM+CFU-M) in BM, without any influence of suspension (no hypoxiaxsuspension interaction). Conversely, a significant interaction was found for erythroid progenitors (BFU-E) (Figure 2C). Indeed, hypoxia decreased the BM pool of BFU-E by $86 \%$ in control animals ( $\mathrm{NC}$ group vs $\mathrm{HC}$ group) and suspension decreased it by $55 \%$ in normoxic mice (NS group vs $\mathrm{NC}$ group). However, suspension added onto hypoxia did not lead to a further BFU-E pool reduction, indicating that suspension and hypoxia do not superimpose their effects. Then, we studied whether the medullar depletion of the HSPCs coincided with their mobilization into the peripheral blood. A significant hypoxiaxsuspension interaction $(p<0.05)$ was found for both myeloid and erythroid progenitor cell numbers in blood (Figure $2 \mathrm{~B}$ and $\mathrm{D}$ ). Post-hoc comparisons revealed that hypoxia produced a different effect whether the animals were suspended or not. Hypoxia increased the number of erythroid and myeloid progenitors in control animal blood (NC vs $\mathrm{HC}$ ) by $+97 \%$ and $+92 \%$, respectively. No significant difference was evidenced between NS and HS groups, suggesting that suspension prevents the hypoxia-driven mobilization of hematopoietic progenitor cells in blood.

\section{Suspension does not alter hypoxia-driven hematological changes}

In peripheral blood samples, ANOVA indicated a main hypoxia effect on blood formulation, without any influence of suspension or hypoxiaxsuspension interaction (Table 1). As expected, four days of hypoxia led to a massive rise in reticulocyte counts $(+260.7 \%)$ and significant increases in hematocrit $(+18.4 \%)$, hemoglobin rate $(+18.3 \%)$ and red blood cell counts $(+14.2 \%)$. Hypoxia effects were not 


\section{Bone Marrow (BM)}

A

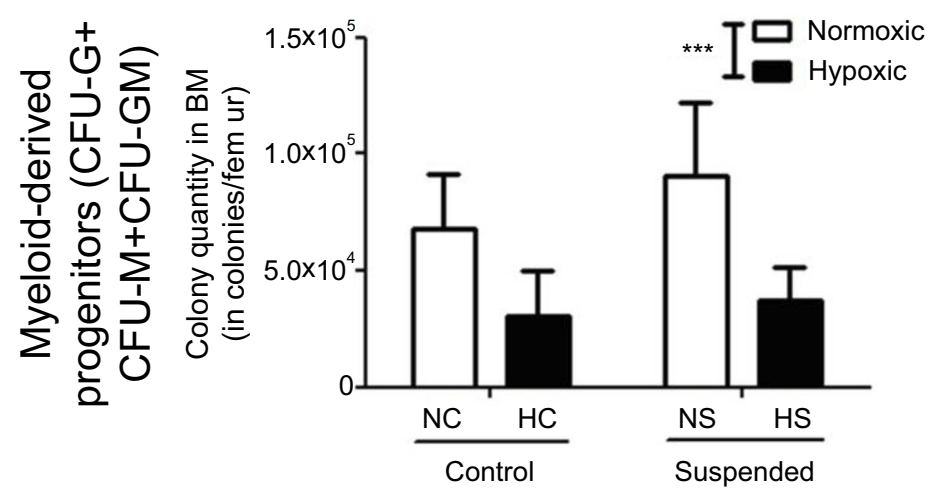

C

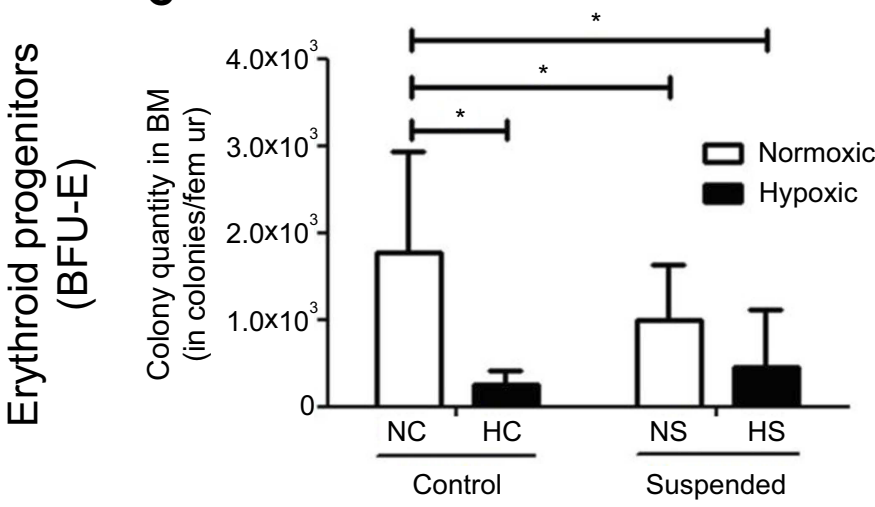

B

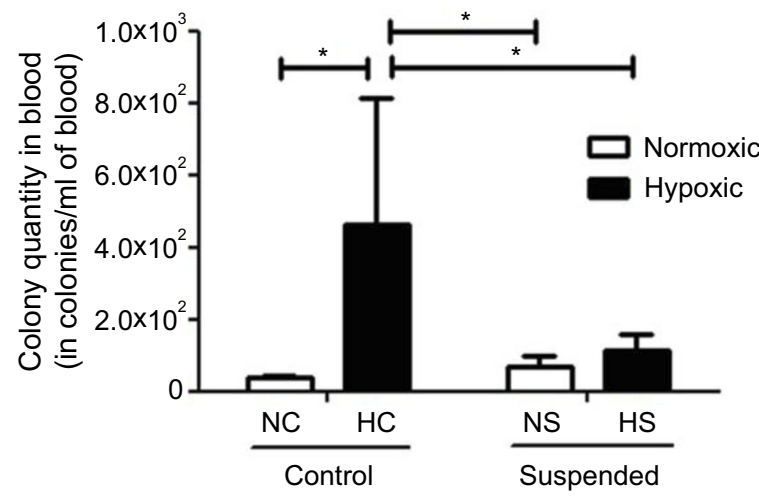

D

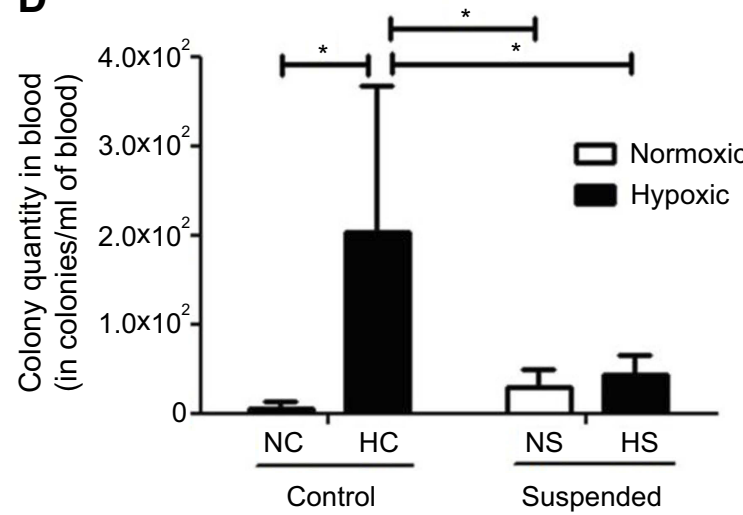

Figure 2 Suspension alters the hypoxia-induced egress of HSPCs from bone marrow to blood. Colony assay was used to determine the content of myeloid-derived progenitors [colony-forming cells sorted out as the number of CFU-granulocyte (CFU-G), CFU-macrophage (CFU-M) and CFU-granulocyte macrophage (CFU-GM)] in bone marrow (BM, panel A) and blood (panel B) of normoxic control (NC), normoxic suspended (NS), hypoxic control (HC) and hypoxic suspended (HS) animals ( $\mathrm{n}=\mathrm{I0/}$ group). The content of erythroid progenitors (burst-forming unit-erythroid BFU-E) was similarly determined in bone marrow (BM, panel C) and blood (panel D) of the animals. $*_{p} \leq 0.05, * * * p \leq 0.001$.

restricted to the erythroid lineage, since a 2 -fold increase in the white blood cell counts of hypoxic mice was evidenced compared to normoxic mice, mainly involving monocyte increase $(+63.8 \%$ rise $)$.

\section{Hypoxia modulates bone cell responses to suspension, without altering the suspension-driven bone loss}

We then investigated the consequence of hypoxia added onto a stromal stress, namely suspension, on bone metabolism.

As shown in Figure 3A, ANOVA yielded a hypoxiaxsuspension interaction $(p<0.01)$ for the number of $\mathrm{MSC} /$ osteoprogenitors assayed by CFU-F in BM. Post-hoc comparison revealed that suspension significantly decreased by 1.7-fold the osteoprogenitor pool in normoxic mice (NS group vs NC group). Moreover, hypoxia significantly decreased by 2.5-fold the quantity of MSC/osteoprogenitors (CFU-F-colonies) in control animals (NC group vs $\mathrm{HC}$ group). However, the pool of CFU-F in suspended animals was not significantly decreased by hypoxia (HS group vs $\mathrm{HC}$ and NS groups), indicating that suspension and hypoxia do not superimpose their effects. We next asked the effects of suspension and hypoxia on MSCderived bone-forming osteoblasts (Figure $3 \mathrm{~B}$ and $\mathrm{E}$ ). ANOVA yielded a significant hypoxiaxsuspension interaction $(p<0.01)$ for the metaphyseal Ob.S/BS. Likewise with the CFU-F data, post-hoc comparison revealed that the osteoblast surface was decreased by hypoxia in control animals $(-50 \%)$ and by suspension in normoxic animals $(-34 \%)$. However, osteoblast surface in HS animals was significantly not different when compared with HC and NS mice, also showing that suspension and hypoxia do not superimpose their effects. Regarding the bone-resorbing osteoclasts, deriving from the monocyte/macrophage 


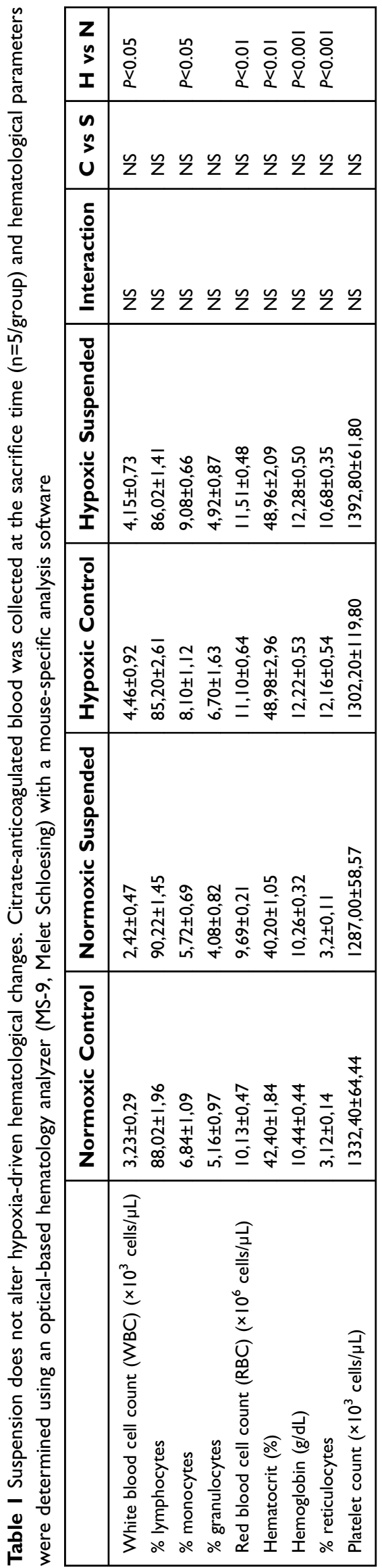

hematopoietic lineage, a significant hypoxiaxsuspension interaction $(p<0.05)$ was found, as shown in Figure 3C. In control animals ( $\mathrm{NC}$ vs $\mathrm{HC}$ ), hypoxia drastically increased Oc.N/BS by 2.0 fold (Figure 3F). While suspension also increased Oc.N/BS in the normoxic environment (NC vs NS animals), the suspension-triggered rise was canceled out in the hypoxic environment ( $\mathrm{HC}$ vs $\mathrm{HS}$ ). Changes in bone mass representing the effect of both bone formation and bone resorption, thereby we examined trabecular bone volume fractions (BV/TV, Figure 3D). A main suspension effect $(p<0.05)$ was detected for this parameter without hypoxiaxsuspension interaction, although such an interaction was noticed for both Ob.S/ BS and Oc.N/BS.

\section{Suspension and hypoxia interact at the molecular level}

To initiate understanding in underlying mechanisms contributing to the different interactions seen at the BM level, cytokine multiplex analysis was performed in our mice BM supernatants. We focused on molecular factors known to be involved in the regulation of hematopoiesis or bone metabolism into the BM environment. In addition, these molecules have previously been shown to be impacted by hypoxia and suspension in mice bearing bone defect. ${ }^{18}$ Interestingly, different patterns of changes were detected upon hypoxia and suspension challenges, as shown in Figure 4.

The levels of IGF-1 and VEGF in the BM supernatants were significantly downregulated $(p<0.01$ and $p<0.001$ for IGF-1 and VEGF, respectively) by the hypoxic challenge (NC+NS vs $\mathrm{HC}+\mathrm{HS}$ groups). Neither effect of suspension nor hypoxiaxsuspension interaction was revealed on these cytokine levels. By contrast, a significant interaction between hypoxia and suspension was evidenced for other factors such as HGF $(p<0.01)$, SDF-1 $(p<0.01)$ and MIP$1 \alpha(p<0.05)$. HGF level in BM supernatants is significantly decreased by $55 \%$ by hypoxia in control animals (NC group vs $\mathrm{HC}$ group) and by $60 \%$ by suspension in normoxic mice (NS group vs NC group). However, in suspended animals, additional hypoxia failed to decrease even more the level of HGF (HS group vs NS group). MIP- $1 \alpha$ was decreased by hypoxia ( $-56 \%)$ in control animals only (NC vs HC) but not in suspended ones (NS vs HS). SDF-1 level was found to be reduced under suspension in the normoxic context (NS vs NC) and also under hypoxia in control animals ( $\mathrm{NC}$ vs $\mathrm{HC}$ ), without any additive effect 
A

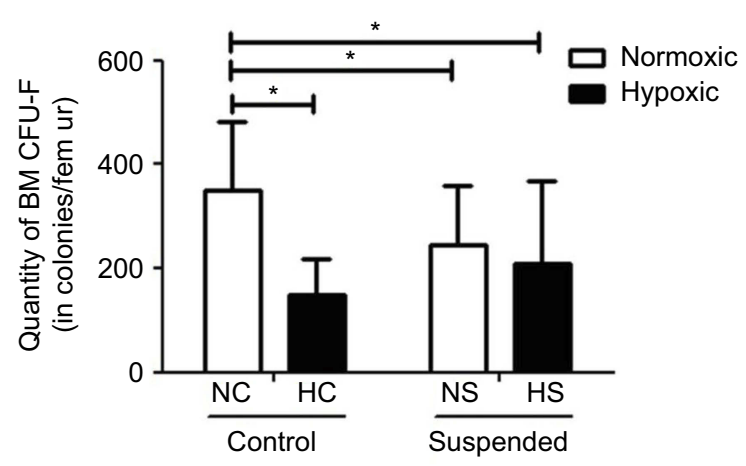

B

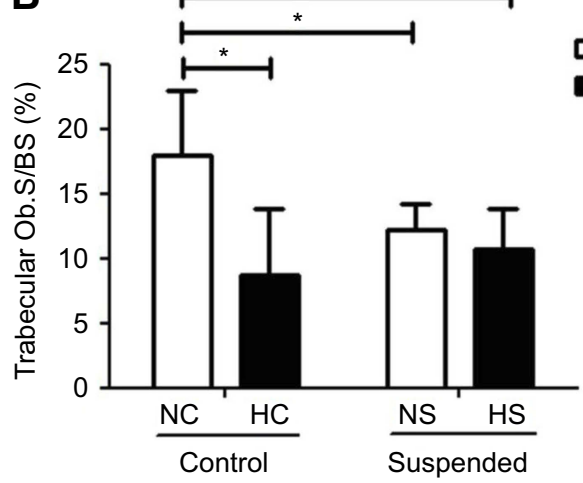

Normoxic

Hypoxic

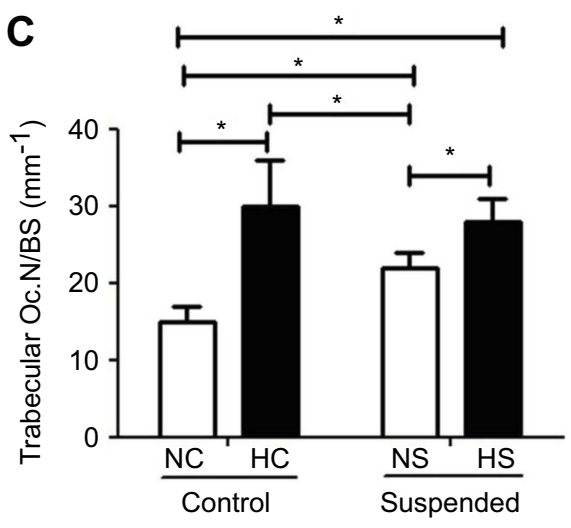

E

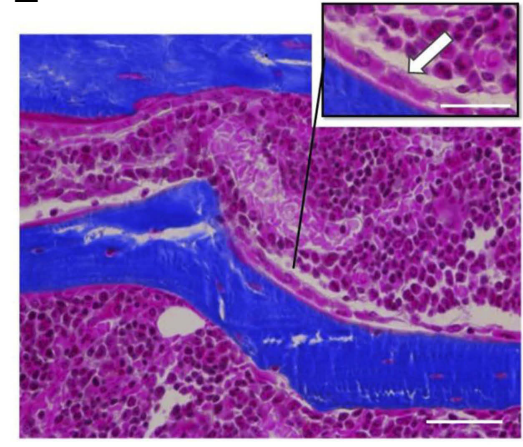

D

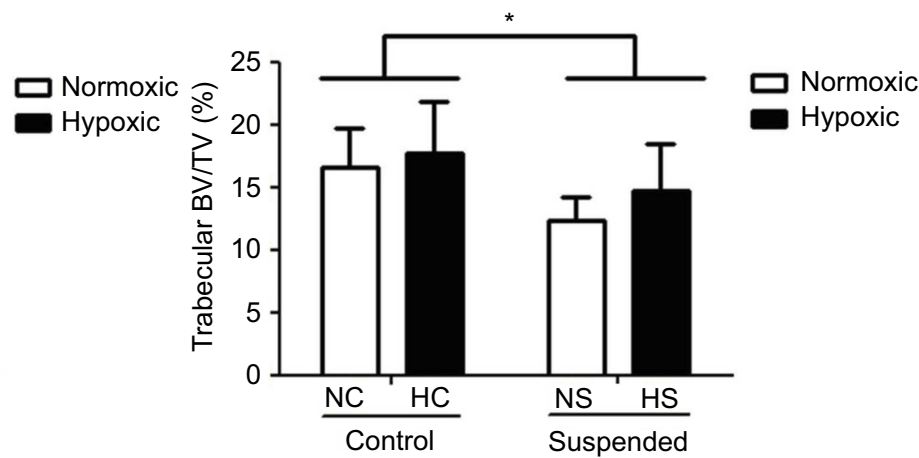

F

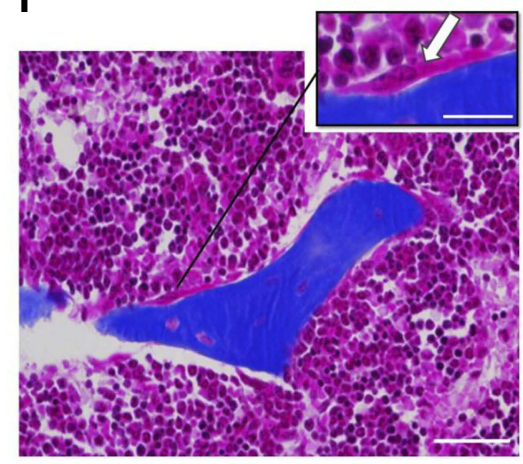

Figure 3 Hypoxia modulates bone cell responses to suspension, without altering the suspension-driven bone loss. (A) Clonogenic capacity was assessed by colonyforming unit-fibroblast (CFU-F) assay in bone marrow (BM) of normoxic control (NC), normoxic suspended (NS), hypoxic control (HC) and hypoxic suspended (HS) animals ( $n=10 /$ group). (B) Histomorphometric quantification of osteoblast surface normalized to bone surface (Ob.S/BS). (C) Histomorphometric quantification of osteoclast number normalized to bone surface (Oc.N/BS). (D) Quantification of femur bone volume/total volume ratio (BV/TV). (E) Representative Masson's trichrome stained trabeculae of normoxic control femur (NC group) showing bone lining osteoblasts compared to (F) representative trabeculae of hypoxic control femur (HC group) showing osteoclast. Scale bars represent $50 \mu \mathrm{m}$ in the main picture area and $25 \mu \mathrm{m}$ in the zoomed areas. Arrows point out osteoblasts (E) and osteoclast $(\mathbf{F})$. All histomorphometric measurements were performed in the distal femoral metaphysis of the animals (For each experimental group $n=5$ littermate mice). ${ }^{*} p \leq 0.05$.

when both suspension and hypoxia were combined. Aside from these BM quantifications, we also investigated the plasmatic level of erythropoietin (Epo) and found a main effect of hypoxia in the Epo concentration $(+382 \%$ increase under hypoxia).

\section{Discussion}

In this study, we aimed at exploring MSC and HSPC maintenance as well as their further fate under hematopoietic and stromal challenges. To this end, we used a two level factorial protocol to assess 1) the hematopoietic 
A

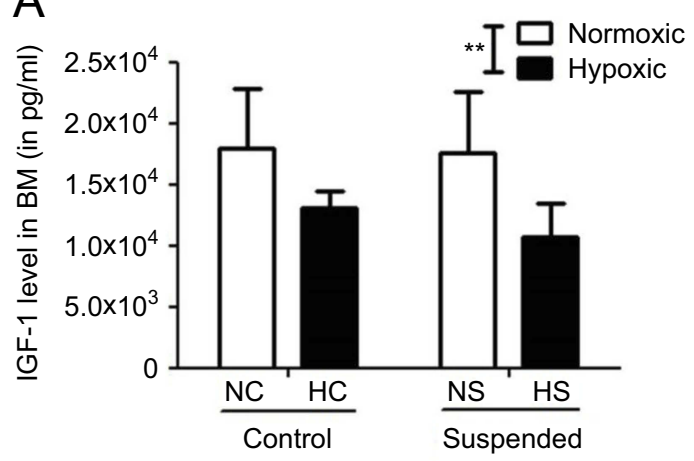

C

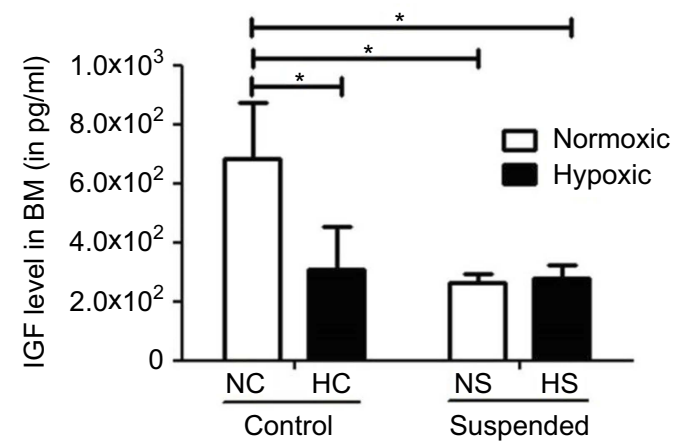

$\mathrm{E}$

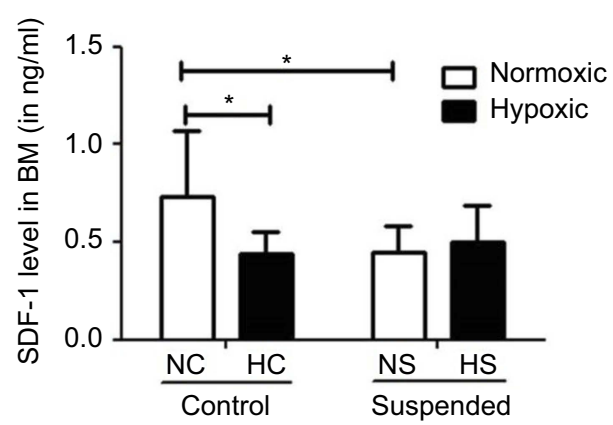

B

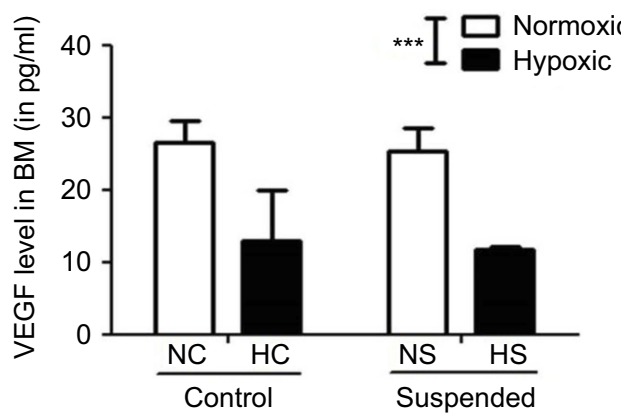

D

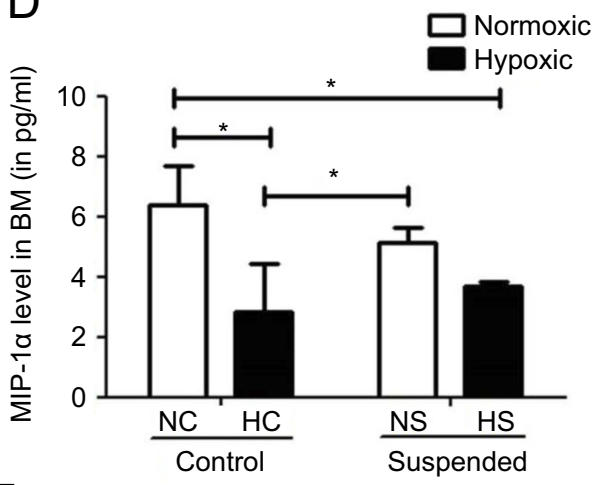

$\mathrm{F}$

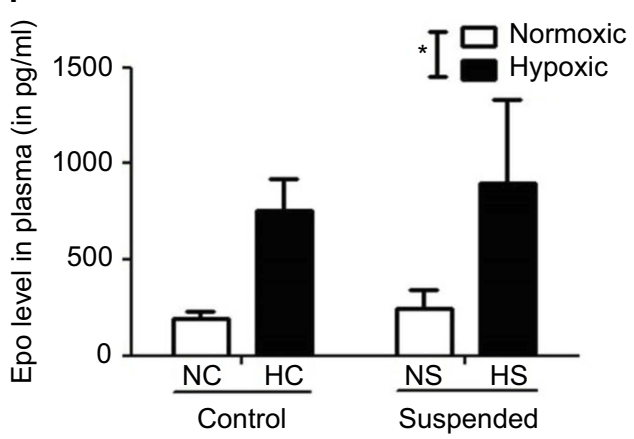

Figure 4 Suspension and hypoxia interact at the molecular level. In BM supernatants, level of proteins involved in stem cell proliferation and mobilization such as (A) IGF-I (insulin-like growth factor-I), (B) VEGF (vascular endothelial growth factor), (C) HGF (hepatocyte growth factor), (D) MIP-I $\boldsymbol{\alpha}$ (macrophage inflammatory protein-I $\boldsymbol{\alpha}$ ), and (E) SDF-I (stromal cell-derived factor-I) was determined using Multiplex Sandwich Elisa (n=5/group) or Elisa kit (for SDF-I only, $n=10 / g r o u p)$. In panel F, plasmatic level of Epo (erythropoietin) was measured by Elisa kit ( $n=5 /$ group). ${ }^{*} p \leq 0.05,{ }^{* *} p \leq 0.0 \mathrm{I}$ and $*^{* * *} p \leq 0.00 \mathrm{I}$.

stress effects per se (hypobaric hypoxia), 2) the stromal stress effects per se (hindlimb suspension) and 3) the combined effects of both challenges.

\section{Responses to hematopoietic challenge per se: hypoxia applied alone without any suspension challenge}

As expected, the most salient feature of low oxygen exposure, namely the hypoxia-induced rise in plasmatic EPO level, was recapitulated in this study. Concomitantly, marked increased hematocrit, hemoglobin rate and red blood cell counts were observed. Although this polycythemic response to hypoxia was expected, the magnitude and the rapid onset of the response after only four days of hypoxic exposure were striking. Presumably, hypoxia-driven polycythemia in our hypoxic animals was intensified by the increased diuresis and reduced plasma proteins usually observed under high-altitude exposure and thus leading to an isotonic hypovolemia. ${ }^{19}$

These profound hematological changes were accompanied by a depletion of the erythroid progenitor pool in BM and its concomitant expansion in the blood microenvironment, suggesting the mobilization of BFU-E progenitors from the BM to the blood circulation. In keeping with this mobilization, SDF-1, a critical regulator of HSPC 
interactions with the osteoblastic niche and a key stem cell mobilizer, was downregulated by hypoxia in the BM. ${ }^{20}$ Regarding bone metabolism, the expectation that hypoxia exposure would hamper osteoblastogenesis is supported by our results, since decreases in CFU-F pool, Ob.S/BS and BM level of pro-osteoblastic factors IGF-1 and HGF were observed under hypoxia. ${ }^{21,22}$ Despite the BM level reduction in pre-osteoclastic factor MIP-1 $\alpha$, the Oc.N/BS increase observed in our hypoxic mice supported previous reports suggesting that in vitro hypoxia triggers osteoclastogenesis. $^{23,24}$ Given the hypoxia-induced increase in bone-resorptive osteoclasts and decrease in bone-forming osteoblasts on trabecular bone surfaces, one would expect a reduced trabecular bone volume fraction in our hypoxic mice. Much to our surprise, we did not observe any bone loss in $\mathrm{HC}$ animals when compared to $\mathrm{NC}$ littermates. A likely explanation to this result might be changes in bone cell activity or that a 4-day hypoxic exposure is not long enough to induce significant changes in bone structure, despite visible changes at bone cell level. Consistently with our results, recent finding from the Plan Hab study also suggests that 21 days of hypoxia in healthy humans do not trigger bone loss. ${ }^{15}$ Likewise, Wang et al report that a 14-day hypoxic exposure does not appear to affect trabecular bone structure and volume in healthy rats. ${ }^{25}$ Thus, whether or not systemic hypoxia exposure drives bone loss is not clear. Another unexpected result was the hypoxia-induced decrease in BM VEGF level. Indeed, hypoxia is known to be a stimulatory factor for VEGF expression in vivo and exposing mice to systemic hypoxia leads to VEGF induction in brain, testis, lung, heart and liver. But conversely, cells at sites of constitutive VEGF expression in normal adult tissues, such as epithelial cells in the choroid plexus and kidney glomeruli, decrease their VEGF expression in response to a systemic hypoxic stimulus. ${ }^{26}$ Since the BM is an established site of constitutive VEGF expression, it is therefore not that surprising to observe in this study a VEGF reduction under hypoxic challenge.

\section{Responses to stromal challenge per se: suspension applied alone without any hypoxic challenge}

The most salient features of suspension-related bone metabolism changes were recapitulated in this study, namely bone loss resulting from a decreased level of bone-forming osteoblasts along with an increased level of bone-resorbing osteoclasts. ${ }^{11,27-31}$ In keeping with the decreased Ob.S/BS, reductions in the osteoprogenitor pool (CFU-F) and BM level of pro-osteoblastic factor HGF were observed under suspension challenge. By contrast, BM IGF-1 level remained unchanged under suspension, although this cytokine may be involved in the process of suspension-related bone loss as previously described. ${ }^{32}$ Regarding hematopoiesis, our findings indicated that suspension reduced the BM level of the hematopoietic stem cell mobilizer factor SDF-1/CXCL-12, in line with previous study. ${ }^{33}$ Moreover, suspension also significantly decreased BFU-E pool size. This reduction in the BM did not coincide with an expansion of the BFU-E progenitors neither in blood or spleen (data not shown in spleen). Alternatively, blood parameters were not affected by suspension despite a faint however not significant decline in red blood cell count and hematocrit. Taken together, these findings suggest that the BM depletion of erythroid progenitors was not related to BFU-E mobilization from BM to blood, but more likely to an underlying anemia which had not yet led to any apparent modification at the blood level by the time of the sacrifice. Indeed, previous report from MoreyHolton indicates that hindlimb suspension in rodents is associated with erythropoiesis inhibition which may result from lowered oxygen requirements due to musculoskeletal unloading. ${ }^{8}$ Lack of suspension effect on the myeloid progenitors in the BM, blood and spleen (data not shown in spleen) reinforced the view that suspension did not trigger HSPC mobilization. ${ }^{8}$

Taken together, our results presented earlier showed that when applied separately both suspension and hypoxic challenges led to bone imbalance caused by an increase in bone-resorbing osteoclasts and a decrease in bone-forming osteoblasts. Though, only the hypoxia-driven bone imbalance appeared to be associated with HSPC mobilization from BM to blood.

\section{Combined effects of hypoxia and suspension}

Previous results from the Plan Hab study in humans refute the idea of a strong modulatory effect of hypoxia on disuse-driven bone loss. ${ }^{15}$ In line with this, we found that the suspension-driven loss in bone volume fraction (BV/ TV) was not impacted by hypoxia. Likewise, changes in hematological parameters in response to hypoxia were not influenced by suspension challenge. However, at the BM level, modulatory effects of interaction between hypoxia and suspension were detected as hypothesized on different cell populations, including BFU-E progenitors. Indeed, our 
data indicated that suspension compromised the hypoxiatriggered egress of erythroid progenitors from $\mathrm{BM}$ to blood. Given the underpinning anemia detected in the suspension group, it is likely that suspended animals have a smaller BFU-E pool available for mobilization when exposed to delayed hypoxia compared to their control littermates. Interactions were also detected on osteoclast and osteoblast numbers. Indeed, either hypoxic stress or suspension alone could lead to bone imbalance, but combination of both challenges did not exacerbate bone imbalance. Such similar observations were made on osteoprogenitors (CFU-F) and BM level of both HGF and SDF1 since we found that the effects of hypoxia and suspension on these parameters did not superimpose each other. Altogether, these lines of evidence may suggest that hypoxia and suspension impact different signaling pathways in the BM. As such, these results are compatible with the current view that BM environment is a highly complex, integrated ensemble of multiple microniches and subtypes of stromal cells each specialized in unique hematopoietic functions and responding differently to stress or injuries. ${ }^{34,35}$ Another key regulatory component in BM niches is the sympathetic nervous system (SNS), which not only regulates physiological homeostatic trafficking of HSPCs but also enables them to respond to acute stress signals. ${ }^{36-38}$ Hypoxia is a potent activator of sympathetic nervous tone, while SNS is also involved in suspensioninduced bone loss. ${ }^{39,40}$ Thus, in our study, it is more likely that suspension and hypoxia responses share signaling pathways, but when both challenges were combined, response amplifications were limited due to physiological thresholds in cellular and molecular parameters.

We are aware that some limitations need to be addressed in our study. First, the onset of the hypoxic episode was delayed by 3 days from the onset of the suspension challenge. This was done for ethical reasons, since we avoided initiating severe procedures simultaneously on mice. Delaying the onsets implies that BM microenvironments were altered by 3 days of suspension prior to triggering the hematopoietic challenge. Findings might have been different upon simultaneous induction of suspension and hypoxia. For instance, suspended mice exposed to delayed hypoxia would not have displayed underlying anemia. Therefore, one could guess that HSPC mobilization would have been less impaired compared to non-suspended hypoxic littermates. Second, it is well documented that the main characteristic of calcium metabolism during spaceflight and in the rodent suspension model is negative calcium balance. ${ }^{28,41-43}$ On another note, hypoxia-induced hyperventilation leads to a further deregulation of acid/base homeostasis and changes in serum calcium. ${ }^{44,45}$ Based on these elements, it would have been interesting to assess interferences in calcium and acid/base balances in this study especially since strong modulation of calcium homeostasis by hypoxia was found in the PlanHab study. ${ }^{15}$

The study described herein is part of a bone repair study in which animals bearing a drill-hole bone defect were exposed to the same experimental set-up combining suspension and delayed hypoxia. ${ }^{18}$ Hypoxia is one of the main trigger of Epo expression. ${ }^{3}$ Consistently, mice challenged with hypoxia in both our present and previous studies displayed high plasmatic levels of EPO. Most recent works point toward a context-dependent role for EPO in the skeletal system. In atraumatic animal models, a bone catabolic effect of EPO is reported. ${ }^{46}$ High plasmatic levels of EPO are associated with a severe trabecular bone loss due to increased bone resorption and decreased bone formation. ${ }^{47-49}$ In contrast, EPO treatment has a bone anabolic effect in animal models of bone regeneration. ${ }^{50-52}$ As discussed earlier, the present study highlighted a catabolic role for hypoxia in bone homeostasis. In a tissue healing context, our previous work showed that a 4-day episode of hypoxia accelerates the healing of a femoral defect. Interestingly, suspension failed to impact the hypoxia-driven enhanced bone repair indicating that hypoxia superimposed its effect on suspension-induced bone resorption.

Taken together, our studies support the assumption of a context-dependent mode of action of Epo on bone physiology, playing opposite skeletal actions during traumatic bone regeneration and steady-state bone remodeling. As such, further studies are warranted to decipher the dual role of EPO on bone metabolism and repair.

\section{Abbreviation list}

$\mathrm{BM}$, bone marrow, MSCs, mesenchymal stromal cells, HSPCs, hematopoietic stem and progenitor cells.

\section{Acknowledgments}

The authors thank Catherine André, Laurent Begot, Dominique Bonneau, Xavier Butigieg, Bernadette Guerton and Sylvie Renault for their valuable technical assistance in our laboratory. The authors are also grateful to Dr. Veronique Chastres for her statistical expertise. This work was supported by a grant from DGA (Délégation Générale pour l'Armement; Grant PDH-1-SMO-0205 to 
Drs. Jean-Jacques Lataillade and Xavier Holy) of the French Ministry of Defence.

\section{Author contributions}

All authors contributed to data analysis, drafting or revising the article, gave final approval of the version to be published, and agree to be accountable for all aspects of the work.

\section{Disclosure}

The authors report no conflicts of interest in this work.

\section{References}

1. Brouard N, Driessen R, Short B, Simmons PJ. G-CSF increases mesenchymal precursor cell numbers in the bone marrow via an indirect mechanism involving osteoclast-mediated bone resorption. Stem Cell Res. 2010;5(1):65-75. doi:10.1016/j.scr.2010.04.002

2. Haase VH. Regulation of erythropoiesis by hypoxia-inducible factors. Blood Rev. 2013;27(1):41-53. doi:10.1016/j.blre.2012.12.003

3. Hiram-Bab S, Neumann D, Gabet Y. Erythropoietin in bone - controversies and consensus. Cytokine. 2017;89:155-159. doi:10.1016/j. cyto. 2016.01 .008

4. Bozzini C, Champin GM, Alippi RM, Bozzini CE. Static biomechanics in bone from growing rats exposed chronically to simulated high altitudes. High Alt Med Biol. 2013;14(4):367-374. doi:10.1089/ ham.2013.1038

5. Bozzini C, Olivera MI, Huygens P, Alippi RM, Bozzini CE. Long-term exposure to hypobaric hypoxia in rat affects femur cross-sectional geometry and bone tissue material properties. Ann Anat Anat Anz Off Organ Anat Ges. 2009;191(2):212-217. doi:10.1016/j.anat.2008.11.002

6. Shang $\mathrm{P}$, Zhang J, Qian A, et al. Bone cells under microgravity. $J$ Mech Med Biol. 2013;13(05):1340006. doi:10.1142/S021951941340006X

7. Grimm D, Grosse J, Wehland M, et al. The impact of microgravity on bone in humans. Bone. 2016;87(SupplementC):44-56. doi:10.1016/j. bone.2015.12.057

8. Morey-Holton E, Globus RK, Kaplansky A, Durnova G. The hindlimb unloading rat model: literature overview, technique update and comparison with space flight data. Adv Space Biol Med. 2005;10:7-40.

9. Machwate M, Zerath E, Holy X, et al. Skeletal unloading in rat decreases proliferation of rat bone and marrow-derived osteoblastic cells. Am J Physiol. 1993;264(5 Pt 1):E790-E799.

10. Sakata T, Sakai A, Tsurukami H, et al. Trabecular bone turnover and bone marrow cell development in tail-suspended mice. J Bone Miner Res Off J Am Soc Bone Miner Res. 1999;14(9):1596-1604. doi:10.1359/jbmr.1999.14.9.1596

11. Shahnazari M, Wronski T, Chu V, et al. Early response of bone marrow osteoprogenitors to skeletal unloading and sclerostin antibody. Calcif Tissue Int. 2012;91(1):50-58. doi:10.1007/s00223-012-9610-9

12. Sonnenfeld G. The immune system in space and microgravity. Med Sci Sports Exerc. 2002;34(12):2021-2027. doi:10.1249/01. MSS.0000039073.04569.B5

13. Cogoli A. Gravitational physiology of human immune cells: a review of in vivo, ex vivo and in vitro studies. $J$ Gravitational Physiol $J$ Int Soc Gravitational Physiol. 1996;3(1):1-9.

14. Bodkin DK, Escalera P, Bocam KJ. A human lunar surface base and infrastructure solution. Space Am Inst Aeronaut Astronaut. 2006;19:2006.

15. Rittweger J, Debevec T, Frings-Meuthen P, et al. On the combined effects of normobaric hypoxia and bed rest upon bone and mineral metabolism: results from the PlanHab study. Bone. 2016;91:130-138. doi:10.1016/j.bone.2016.07.013
16. Keramidas ME, Mekjavic IB, Kölegård R, Choukèr A, Strewe C, Eiken O. PlanHab: hypoxia counteracts the erythropoietin suppression, but seems to exaggerate the plasma volume reduction induced by 3 weeks of bed rest. Physiol Rep. 2016;4:7. doi:10.14814/phy2.12760

17. Holy $X, Z$ Zerath $E$. The suspension device for hindlimb unloading in the ratresults of a video monitoring study. Stal.1996;21(3-4):177-184.

18. Durand M, Collombet J-M, Frasca S, et al. In vivo hypobaric hypoxia performed during the remodeling process accelerates bone healing in mice. Stem Cells Transl Med. 2014;3(8):958-968. doi:10.5966/ sctm.2013-0209

19. Honig, A. Body fluid and energy metabolism at high altitude. In: Fregly MJ, Blatteis CM, editors. Handbook of Physiology. Oxford: Oxford University Press; 1996:1277-1289.

20. Petit I, Szyper-Kravitz M, Nagler A, et al. G-CSF induces stem cell mobilization by decreasing bone marrow SDF-1 and up-regulating CXCR4. Nat Immunol. 2002;3(7):687-694. doi:10.1038/ni813

21. Xian L, Wu X, Pang L, et al. Matrix IGF-1 regulates bone mass by activation of mTOR in mesenchymal stem cells. Nat Med. 2012;18 (7):1095-1101. doi:10.1038/nm.2793

22. Aenlle KK, Curtis KM, Roos BA, Howard GA. Hepatocyte growth factor and 38 promote osteogenic differentiation of human mesenchymal stem cells. Mol Endocrinol Baltim Md. 2014;28(5):722-730. doi:10.1210/me.2013-1286

23. Utting JC, Flanagan AM, Brandao-Burch A, Orriss IR, Arnett TR. Hypoxia stimulates osteoclast formation from human peripheral blood. Cell Biochem Funct. 2010;28(5):374-380. doi:10.1002/cbf.1660

24. Arnett TR, Gibbons DC, Utting JC, et al. Hypoxia is a major stimulator of osteoclast formation and bone resorption. $J$ Cell Physiol. 2003;196(1):2-8. doi:10.1002/jcp.10321

25. Wang G, Wang J, Sun D, et al. Short-term hypoxia accelerates bone loss in ovariectomized rats by suppressing osteoblastogenesis but enhancing osteoclastogenesis. Med Sci Monit Int Med J Exp Clin Res. 2016;22:2962-2971. doi:10.12659/MSM.899485

26. Marti HH, Risau W. Systemic hypoxia changes the organ-specific distribution of vascular endothelial growth factor and its receptors. Proc Natl Acad Sci USA. 1998;95(26):15809-15814. doi:10.1073/ pnas.95.26.15809

27. Halloran BP, Bikle DD, Wronski TJ, Globus RK, Levens MJ, MoreyHolton E. The role of 1,25-dihydroxyvitamin D in the inhibition of bone formation induced by skeletal unloading. Endocrinology. 1986;118(3):948-954. doi:10.1210/endo-118-3-948

28. Dehority W, Halloran BP, Bikle DD, et al. Bone and hormonal changes induced by skeletal unloading in the mature male rat. $\mathrm{Am} J$ Physiol. 1999;276(1 Pt 1):E62-E69.

29. David V, Lafage-Proust M-H, Laroche N, Christian A, Ruegsegger P, Vico L. Two-week longitudinal survey of bone architecture alteration in the hindlimb-unloaded rat model of bone loss: sex differences. $\mathrm{Am}$ J Physiol Endocrinol Metab. 2006;290(3):E440-E447. doi:10.1152/ ajpendo.00293.2004

30. Sakai A, Nakamura T. Changes in trabecular bone turnover and bone marrow cell development in tail-suspended mice. $J$ Musculoskelet Neuronal Interact. 2001;1(4):387-392.

31. Wronski TJ, Morey ER. Skeletal abnormalities in rats induced by simulated weightlessness. Metab Bone Dis Relat Res. 1982;4(1):69-75.

32. Long RK, Nishida S, Kubota T, et al. Skeletal unloading-induced insulin-like growth factor 1 (IGF-1) nonresponsiveness is not shared by platelet-derived growth factor: the selective role of integrins in IGF-1 signaling. J Bone Miner Res Off J Am Soc Bone Miner Res. 2011;26(12):2948-2958. doi:10.1002/jbmr.511

33. Visigalli D, Strangio A, Palmieri D, Manduca P. Hind limb unloading of mice modulates gene expression at the protein and mRNA level in mesenchymal bone cells. BMC Musculoskelet Disord. 2010;11:147. doi:10.1186/1471-2474-11-147

34. Yu VWC, Scadden DT. Heterogeneity of the bone marrow niche. Curr Opin Hematol. 2016;23(4):331-338. doi:10.1097/MOH.00000000 00000265 
35. Birbrair A, Frenette PS. Niche heterogeneity in the bone marrow. Ann N Y Acad Sci. 2016;1370(1):82-96. doi:10.1111/nyas.13016

36. Yamazaki S, Ema H, Karlsson G, et al. Nonmyelinating Schwann cells maintain hematopoietic stem cell hibernation in the bone marrow niche. Cell. 2011;147(5):1146-1158. doi:10.1016/j.cell.2011.09.053

37. Katayama Y, Battista M, Kao W-M, et al. Signals from the sympathetic nervous system regulate hematopoietic stem cell egress from bone marrow. Cell. 2006;124(2):407-421. doi:10.1016/j.cell.2005.10.041

38. Ferraro F, Lymperi S, Méndez-Ferrer S, et al. Diabetes impairs hematopoietic stem cell mobilization by altering niche function. Sci Transl Med. 2011;3(104):104ra101. doi:10.1126/scitranslmed.3002191

39. Richalet JP, Larmignat $P$, Rathat $C$, Kéromès A, Baud P, Lhoste F. Decreased cardiac response to isoproterenol infusion in acute and chronic hypoxia. J Appl Physiol Bethesda Md 1985. 1988;65 (5):1957-1961. doi:10.1152/jappl.1988.65.5.1957

40. Kondo H, Nifuji A, Takeda S, et al. Unloading induces osteoblastic cell suppression and osteoclastic cell activation to lead to bone loss via sympathetic nervous system. J Biol Chem. 2005;280(34):3019230200. doi:10.1074/jbc.M504179200

41. Smith SM, McCoy T, Gazda D, Morgan JLL, Heer M, Zwart SR. Space flight calcium: implications for astronaut health, spacecraft operations, and earth. Nutrients. 2012;4(12):2047-2068. doi:10.3390/ nu4122047

42. Heer M, Kamps N, Biener C, et al. Calcium Metabolism in Microgravity. Eur J Med Res. 1999;4(9):357-360.

43. Navidi M, Evans J, Wolinsky I, Arnaud SB. Calcium balance in mature male rats with unloaded hindlimbs. Aviat Space Environ Med. 2004;75(6):483-488.

44. Drummer C, Friedel V, Börger A, et al. Effects of elevated carbon dioxide environment on calcium metabolism in humans. Aviat Space Environ Med. 1998;69(3):291-298.
45. Schaefer KE, Pasquale SM, Messier AA, Niemoeller H. CO2induced kidney calcification. Undersea Biomed Res. 1979;6(Suppl): S143-S153.

46. Hiram-Bab S, Liron T, Deshet-Unger N, et al. Erythropoietin directly stimulates osteoclast precursors and induces bone loss. FASEB J Off Publ Fed Am Soc Exp Biol. 2015;29(5):1890-1900. doi:10.1096/ fj. 14-259085

47. Oikonomidou PR, Casu C, Yang Z, et al. Polycythemia is associated with bone loss and reduced osteoblast activity in mice. Osteoporos Int J Establ Result Coop Eur Found Osteoporos Natl Osteoporos Found USA. 2016;27(4):1559-1568. doi:10.1007/ s00198-015-3412-7

48. Rauner M, Franke K, Murray M, et al. Increased EPO levels are associated with bone loss in mice lacking PHD2 in EPO-producing cells. J Bone Miner Res Off J Am Soc Bone Miner Res. 2016;31 (10):1877-1887. doi:10.1002/jbmr.2857

49. Dewamitta SR, Russell MR, Nandurkar H, Walkley CR. Darbepoietin-alfa has comparable erythropoietic stimulatory effects to recombinant erythropoietin whilst preserving the bone marrow microenvironment. Haematologica. 2013;98(5):686-690. doi:10.3324/haematol.2012.078709

50. Bakhshi H, Kazemian G, Emami M, Nemati A, Karimi Yarandi H, Safdari F. Local erythropoietin injection in tibiofibular fracture healing. Trauma Mon. 2013;17(4):386-388. doi:10.5812/traumamon.7099

51. Betsch M, Thelen S, Santak L, et al. The role of erythropoietin and bone marrow concentrate in the treatment of osteochondral defects in mini-pigs. PLoS One. 2014;9(3):e92766. doi:10.1371/journal. pone.0092766

52. Garcia P, Speidel V, Scheuer C, et al. Low dose erythropoietin stimulates bone healing in mice. J Orthop Res Off Publ Orthop Res Soc. 2011;29(2):165-172. doi:10.1002/jor.21219
Hypoxia

\section{Publish your work in this journal}

Hypoxia is an international, peer-reviewed, open access journal that aims to improve understanding of the biological response to hypoxia. The journal will publish original research articles, reviews, methodological advances, clinical studies, and expert opinions that identify developments in the regulation of the physiological and

Submit your manuscript here: https://www.dovepress.com/hypoxia-journal pathological responses to hypoxia and in the therapeutic targeting of hypoxia-responsive pathways. The manuscript management system is completely online and includes a very quick and fair peer-review system, which is all easy to use. Visit http://www.dovepress.com/ testimonials.php to read real quotes from published authors. 